\title{
In men at risk of HIV infection, IgM, lgG1, lgG3, and IgA reach the human foreskin epidermis
}

\author{
MP Lemos ${ }^{1}$, ST Karuna ${ }^{1}$, GJ Mize ${ }^{1}$, Y Fong ${ }^{1}$, SM Montano $^{2}$, C Ganoza $^{3}$, JR Lama ${ }^{3}$, J Sanchez ${ }^{3}$ and \\ MJ McElrath ${ }^{1,4,5,6}$
}

We profiled the humoral response in the penis, an area that has been minimally explored but may be relevant for protecting insertive men against HIV and other sexually acquired infections. Comparing paired tissue samples from 20 men at risk of HIV infection, foreskin contains less immunoglobulin A $(\operatorname{IgA})$ and more $\lg G 2$ than colon. Using foreskin dermal and epidermal explants and paired plasma from 17 men, we examined lg accumulation by normalizing lg to human serum albumin (HSA) transudation. Dermal IgM, IgG2, IgA, and IgE ratios were greater than that in plasma, suggesting there is local antibody secretion at the dermis. Local Ig transcription was concentrated at the inner rather than the outer foreskin, and inner foreskin Ig ratios did not correlate with blood, indicating that localized production can contribute to the foreskin response. IgM, IgG1, IgG3, and IgA have preferential access to the foreskin epidermis, whereas IgG2, IgG4, and IgE are restricted to the dermis. Lastly, Ad5-specific IgA was selectively present in the colon, whereas foreskin Ad5 IgG was mainly derived from blood, and reached the inner epidermis at higher ratios than the outer $(P<0.002)$. In summary, the foreskin antibody response combines local and systemic sources, and there is selective isotype accumulation in the epidermis.

\section{INTRODUCTION}

Substantial evidence has emerged over the years in non-human primate models that potent antibodies can mediate protective effects against SIV and SHIV infection. ${ }^{1}$ In humans, the RV144 clinical trial demonstrated $31 \%$ protection among Thai volunteers $^{2}$ where infection risk was directly associated with blood immunoglobulin A ( $\operatorname{IgA})$ titers against the $\mathrm{C} 1$ region of HIV Env and inversely associated with high titers of anti-Env V1V2 IgG3. ${ }^{3-5}$ However, it remains to be determined whether these vaccine correlates can protect at the rectal, vaginal, and penile surfaces, where HIV is predominantly transmitted. Whether passively infused antibodies or HIV vaccine candidates can reach human genital and rectal sites at sufficient concentrations is also unclear.

Quantitative methods to measure vaginal and rectal antibody responses have been developed, ${ }^{8}$ but penile Ig assessments are limited. In uncircumcised heterosexual men ${ }^{9-11}$ and men who have insertive sex with men, ${ }^{12,13}$ the foreskin is an important site of HIV exposure, ${ }^{14}$ as three independent randomized controlled trials showed that circumcision reduced HIV infection risk by $51-60 \% .^{9-11}$ However, $70 \%$ of the world's men remain uncircumcised, ${ }^{15}$ and the uptake of circumcision faces logistical ${ }^{16,17}$ and cultural barriers, ${ }^{18}$ so it remains important to investigate whether the foreskin can be armed with humoral responses that can prevent HIV infection. In fact a recent study demonstrated that SIV can preferentially persist in proximity to target cells at the macaque inner foreskin and glands, but not the outer foreskin, ${ }^{14}$ indicating that immune control at these sites may be most useful for protection.

Distinct Ig isotypes predominate in various mucosal surfaces, suggesting that antibody restrictions are present at the sites of host-pathogen interactions. IgM is first induced during the immune response to a new antigen and has $\mathrm{Clq}$ and complement activation functions; however, it is present in low concentrations in the female genital and intestinal surfaces, and is undetectable in seminal fluid of most

\footnotetext{
${ }^{1}$ Vaccine and Infectious Disease Division, Fred Hutchinson Cancer Research Center, Seattle, Washington, USA. ${ }^{2}$ US Naval Medical Research Unit 6 (NAMRU-6), Lima, Perú. ${ }^{3}$ Asociación Civil Impacta Salud y Educación, Lima, Perú. ${ }^{4}$ Department of Medicine, University of Washington, Seattle Washington, USA. ${ }^{5}$ Department of Laboratory Medicine, University of Washington, Seattle Washington, USA and ${ }^{6}$ Department of Global Health, University of Washington, Seattle Washington, USA. Correspondence: MJ McElrath (jmcelrat@fhcrc.org) 
healthy men. ${ }^{19-21} \mathrm{IgA}$ isotypes dominate in the intestine and can inactivate pathogens by neutralization and exclusion. ${ }^{21-23}$ Both intestinal and genital IgA rely on local production, with minor components transudating from blood. ${ }^{24-28}$ Compared with other isotypes, IgG is most abundant in blood, semen, cervical, and vaginal compartments. ${ }^{29}$ There are four subclasses arranged by their abundance in serum: IgG1, IgG2, IgG3, and IgG4. They have remarkable differences in complement activation, phagocytosis, antibody-dependent cell-mediated cytotoxicity, and Fc-Receptor binding, with a general order of activating capacity being $\operatorname{IgG} 3>\operatorname{IgG} 1 \gg \operatorname{IgG} 2>\operatorname{IgG} 4 .{ }^{30}$

In addition to the isotypes, the specificity of the antibody response can also be compartmentalized. Exposure to oral Salmonella typhi or intranasal adenovirus can lead to IgG antibody responses that concentrate in the nose and mouth, as well as vaginal IgA; ${ }^{31,32}$ whereas rectal exposure can lead to antigen-specific IgG in tears and IgA that dominates in rectal secretions. ${ }^{31}$ Deltoid delivery of a canarypox HIV vaccine can generate both IgG and IgA in rectal secretions, but this is limited after inguinal immunizations, which drain the genitals. ${ }^{33}$ Thus, the immunization strategies and natural infections that trigger penile antibody responses may not match those that successfully generate responses at mucosal surfaces.

To better understand the antibody profile that may have a role in controlling infections at the foreskin, we evaluated the humoral responses in the foreskin of sexually active young men who have sex with men at high risk of HIV infection, and compared these with colonic and systemic B-cell responses. Our results indicate that some foreskin Ig isotypes transudate directly from blood, whereas others are locally produced. These findings have important implications for the development of strategies to induce relevant Ig responses against sexually transmitted infections (STIs) so that immune responses reach this important site of pathogen exposure.

\section{RESULTS}

\section{Foreskin tissue has different Ig isotype distributions than} colon tissue

To explore the tissue Ig isotype distributions in sexually active men, we generated protein lysates and compared Ig concentrations from 20 matched colonic and foreskin tissues (including inner and outer foreskin), normalized for protein content (Figure 1). No differences were detected in total IgA or IgGs when men with STIs were compared with men without STIs (data not shown), so they were studied as a single cohort. The median IgA concentration was at least fourfold greater in the colon (3.13 $\mathrm{ng} \mathrm{\mu g}^{-\mathrm{g}}$, interquartile range (IQR) 1.34-4.93) than in the inner $\left(0.72 \mathrm{ng} \mathrm{\mu g}^{-1}\right.$, IQR $\left.0.45-2.17 ; P=0.001\right)$ and outer foreskin tissue $\left(0.65 \mathrm{ng} \mathrm{gg}^{-1}\right.$, IQR $0.38-2.82 ; P=0.001$; Figure 1a), and at least twofold greater than that in blood $(P=0.001$; data not shown $)$.

IgG1 had the highest overall median concentration in all tissues and there were no significant differences among them (Friedman Test $P=0.387$; Figure 1b). Interestingly, colonic tissue median IgG2 concentrations (1.24 ng $\mu \mathrm{g}^{-1}$, IQR 0.19-
2.51) were lower compared with inner $\left(2.36 \mathrm{ng}_{\mu \mathrm{g}}{ }^{-1}\right.$, IQR $0.30-4.73 ; P=0.011)$ and outer foreskin $\left(2.85 \mathrm{ng} \mathrm{\mu g}^{-1}\right.$, IQR 1.00-3.88; $P=0.001$ (Figure 1c). IgG3 and IgG4 were less abundant, and comparable among the different tissues (Friedman $P=0.951$ and $P=0.981$, respectively; Figure 1d, e). These colon and foreskin tissue IgG to protein ratios are $5.6-11.8 \%$ of those expected in plasma (data not shown). The results indicated that, similar to the female genital tract, ${ }^{26,34}$ foreskin has a unique distribution of antibody isotypes that are distinct from intestinal tissues.

\section{Foreskin sources of antibody combine transudation from blood and local production}

Antibodies might reach the foreskin through convection/ transudation from blood, local production, or both. To define the sources of Igs in the foreskin of sexually active men, we separately cultured dermal and epidermal explants from 20 donors, measured Ig/HSA ratios released into 48 -h culture supernatants, and compared levels of released Igs with those in paired blood taken prior to the procedure. Three participants' tissues were excluded because of inefficient dermal-epidermal separation, as indicated by hemoglobin contamination (data not shown). In the remaining 17 individuals, dermal Ig/HSA ratios were comparable with or higher than plasma (Figure 2). Foreskin IgA/HSA ratios at the inner dermis and the outer dermis were increased sixfold $(P<0.001)$ and threefold $(P=0.008)$, respectively, when compared with plasma (Figure 2a). Inner foreskin IgG2/HSA ratios were fivefold larger than blood ratios $(P=0.009$; Figure 2c). Foreskin inner dermis IgM/HSA ratios and outer dermis IgM/HSA ratios were increased by threefold $(P<0.001)$ and twofold $(P=0.030)$ compared with plasma (Figure 2f). Similarly, inner $(P<0.001)$ and outer dermis IgE/HSA ratios $(P<0.001)$ were greater than in plasma (Figure 2g). Thus, the results suggested local accumulation or production of most Ig isotypes at the dermal foreskin, after $48 \mathrm{~h}$ of culture.

To determine whether Ig transudation from blood is a primary contributor to humoral responses at the foreskin dermis, we examined the correlation of plasma Ig/HSA ratios with inner and outer foreskin dermis ratios (Figure 3). No correlation was identified among the inner dermal isotypes and blood (Figure 3, black circles), supporting that antibody is locally produced/accumulated at the inner dermis. Similar results were found for outer foreskin dermal IgA, IgG1, IgG2, and IgM and their blood counterparts (Figure 3a-c, f), suggesting that dominant isotypes have local production/accumulation at the foreskin. However, the concentrations of less frequent Ig isolates-IgG3, IgG4, and IgE-were directly correlated between outer foreskin and blood (IgG3: $r=0.544, P=0.013$ (Figure 3d); IgG4: $r=0.719, P=0.0004$ (Figure 3e); IgE: $r=0.568, P=0.009$ (Figure 3g)). Thus, these results suggest that blood sources are the major contributors of outer foreskin IgG3, IgG4, and IgE antibodies, whereas other Igs have localized foreskin accumulation or production that contributes to the total response. 
a

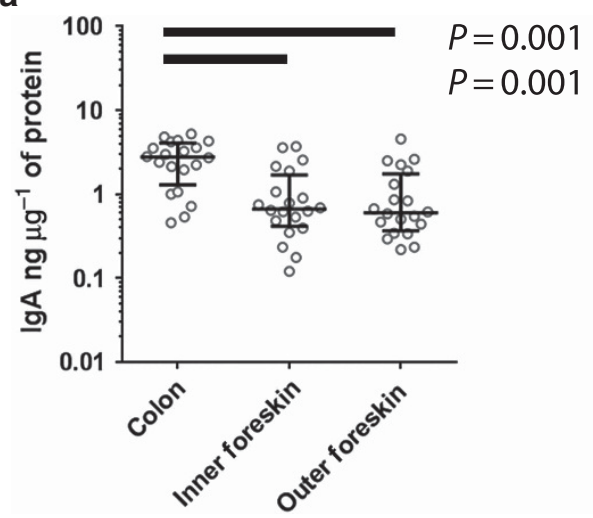

C

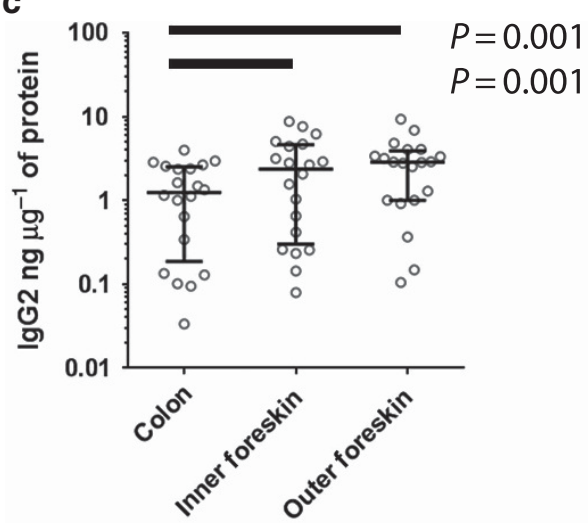

b

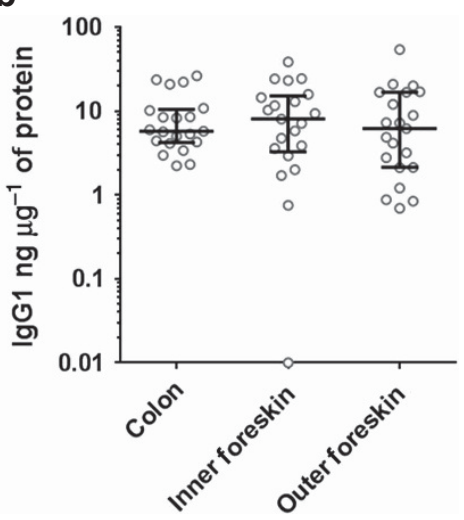

d

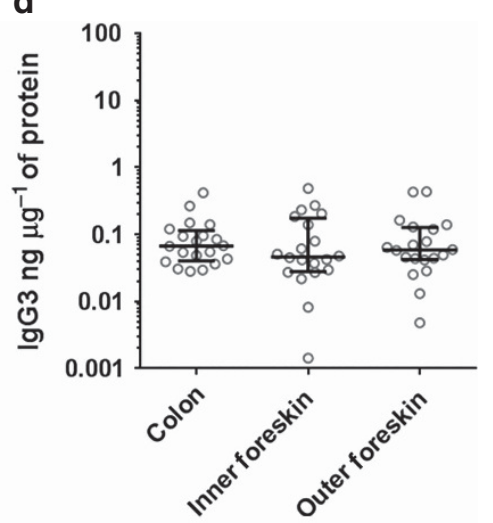

e

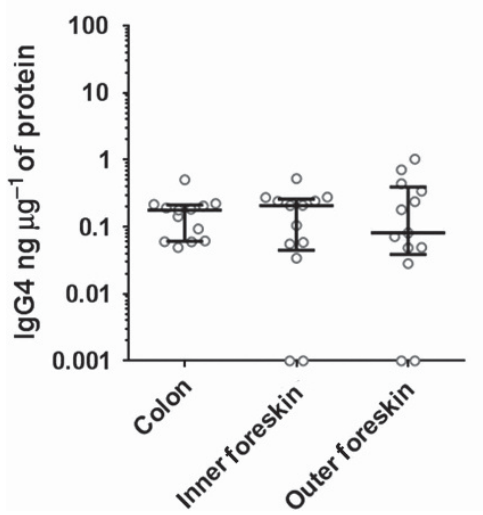

Figure 1 The foreskin contains more lgG2 and less IgA than colonic mucosa. Twenty paired foreskin and colonic tissue samples were homogenized and assayed by multiplex bead array for (a) IgA, (b) $\lg G 1$, (c) $\lg G 2$, (d) $\lg G 3$, and (e) $\lg G 4$. All Ig concentrations were normalized to microgram of protein in the tissue biopsy. Bars indicate median with IQR for each tissue. Lysate buffer designed for homogenization of skin samples prevented detection of IgM and $\mathrm{IgE}$ standards, and thus their quantitation was not included in the figure. Only significant differences in Wilcoxon post-test after Bonferroni correction for three comparisons are reported in the figure. Ig, immunoglobulin; IQR, interquartile range.

\section{Foreskin dermis contains IgM-, IgG-, and IgA-secreting cells, but no organized B-cell follicles}

To determine whether local antibody-secreting cells (ASCs) could be producing foreskin Igs, we first investigated the evidence for local transcription of Ig genes, comparing inner and outer foreskin RNA samples with matched samples of blood and colon. A primer capable of amplifying IgM, IgG1, IgG3, and IgG4 transcripts (IgMG134; Figure 4a) or IgA
(Figure 4b) using real-time PCR identified ASCs in all tissues in different frequencies. The results were normalized to an endogenous control, TATA-box binding protein (TBBP). Median relative expression of colonic IgMG134 (3.189, IQR 3.189-5.348) was higher compared with blood (1.381, IQR $0.846-2.315 ; P=0.0001)$, inner $(0.038$, IQR $0.010-0.240$; $P<0.0001$ ), and outer foreskin (0.002, IQR 0.001-0.024; $P<0.0001)$. Median relative expression of colon IgA 
a

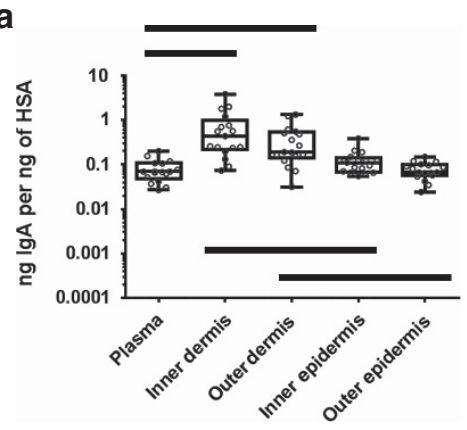

C

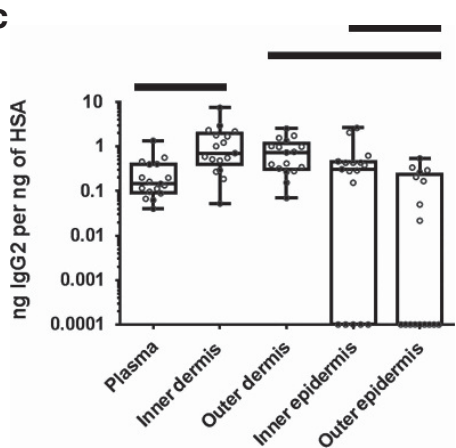

e
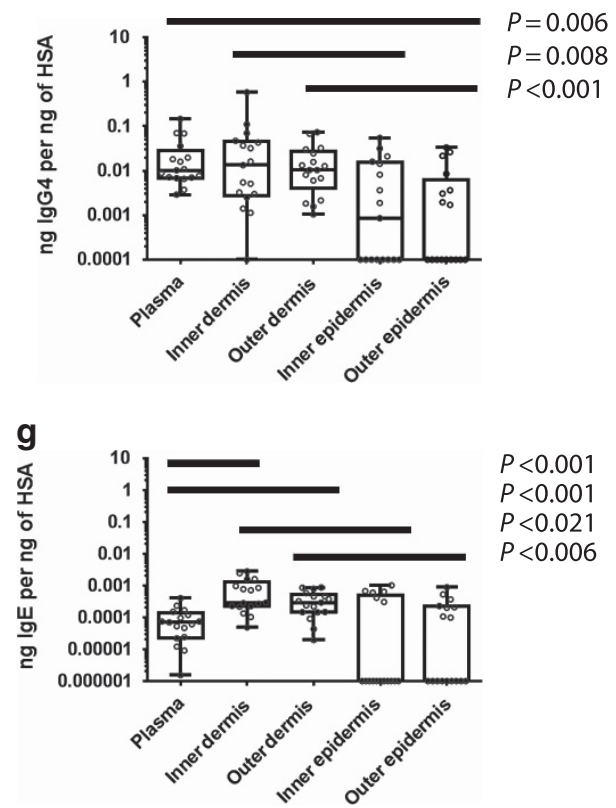

b

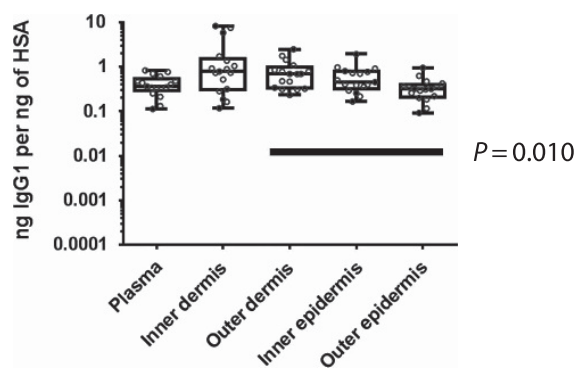

d

$P=0.009$

$P<0.001$

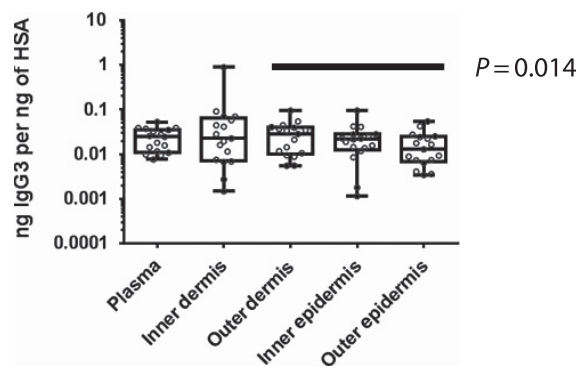

f

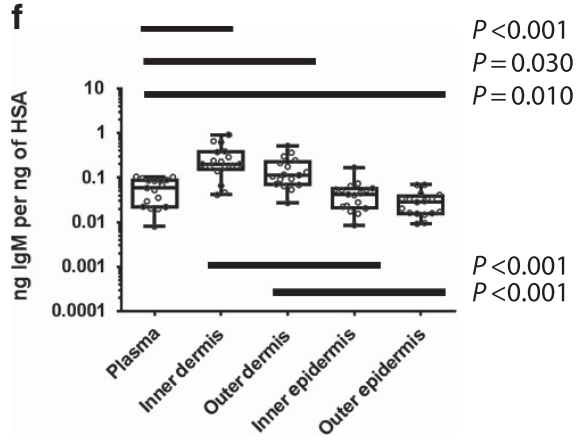

Figure 2 Comparison of dermal and epidermal foreskin Igs with blood Igs. Foreskin and plasma samples from 17 participants were compared by calculating the ratios of each antibody concentration normalized to HSA in each sample. HSA, (a) $\lg A$, (b) $\lg G 1$, (c) $\lg G 2$, (d) $\lg G 3$, (e) $\lg G 4$, (f) $\lg M$, and (g) IgE were measured by MBA. Samples under the level of detection are graphed at the bottom of the axis. Only significant differences in Wilcoxon posttest after Bonferroni correction for eight comparisons are reported in the figure. HSA, human serum albumin; Ig, immunoglobulin; MBA, multiplex bead array.

(51.26, IQR 33.00-97.21) was also increased compared with blood (3.23, IQR 2.45-5.15; $P=0.0001)$, inner (0.041, IQR $0.013-0.273 ; \quad P<0.0001)$, and outer foreskin $(0.011$, IQR $0.002-0.023 ; P<0.0001)$. Blood IgMG134/TBBP and IgA/TBBP ratios were also significantly higher than both foreskin areas $(P<0.0001$ each $)$. The results indicated that foreskin Ig transcription is low, but detectable.

No differences in Ig transcription in colon or foreskin were detected among men with STIs and men without STIs in our cohort (data not shown). Interestingly, there appeared to be 
a

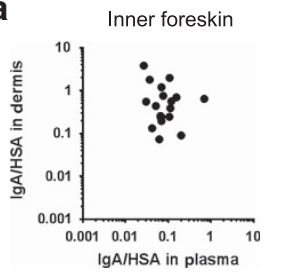

b

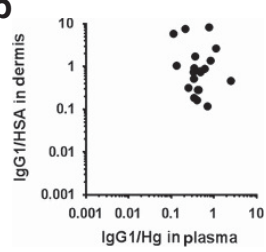

C

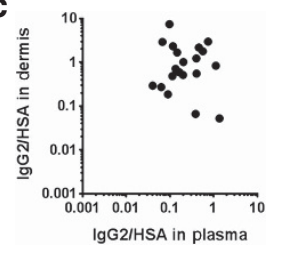

d

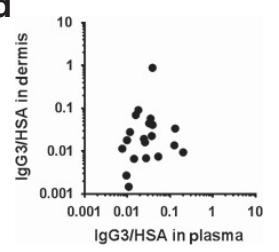

e

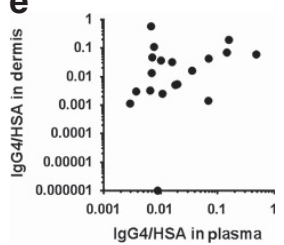

f

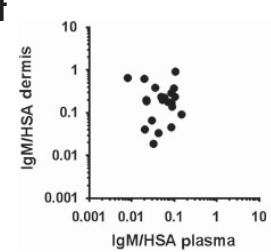

g

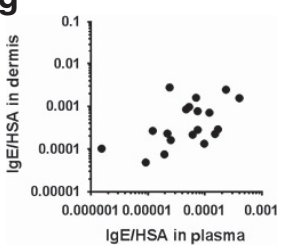

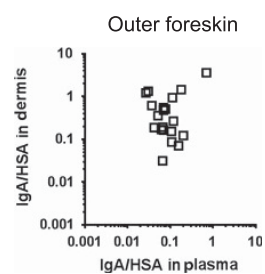
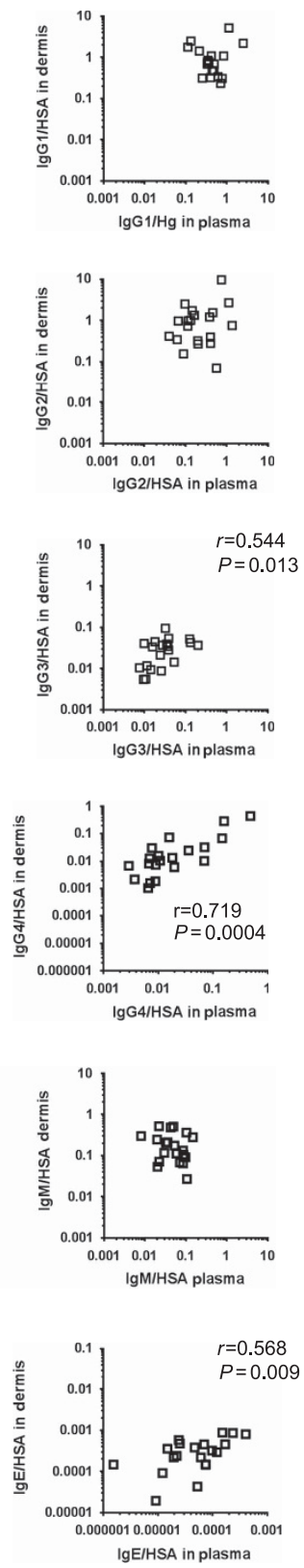

Figure 3 Most Ig/HSA ratios in the foreskin dermis do not correlate with those in blood. Correlation between 17 foreskin dermal antibody HSA ratios and those in paired blood were analyzed using Spearman's Correlation. HSA, (a) IgA, (b) IgG1, (c) IgG2, (d) IgG3, (e) IgG4, (f) $\operatorname{lgM}$, and (g) IgE were measured via MBA. Inner dermis samples are labeled with filled black circles; outer dermis samples are labeled with open black squares. Spearman's $P$ values reported in the figure are shown after Bonferroni correction for seven comparisons. HSA, human serum albumin; Ig, immunoglobulin; MBA, multiplex bead array. compartmentalization of the Ig transcription at the foreskin, with inner foreskin levels being consistently higher than outer (Figure $4 \mathbf{a} ; P<0.0001$ ). Thus, the inner foreskin appears to especially accumulate ASCs capable of local Ig transcription.

To test for the presence of ASCs in the foreskin dermis, we also conducted immunofluorescence microscopy staining of inner and outer foreskin tissues. In healthy men, plasma cells were identified by their co-staining for CD138 and intracellular IgA or IgG. The cells were rare, most often located in dermal areas, and not organized in follicular structures (Figure 4c). Inner foreskin (median 101.2 IgG + or IgA + cells per $\mathrm{mm}^{2}$ IQR 55.5-153.5) contained more cells than the outer (median $67.4 \mathrm{IgG}+$ or IgA + cells per mm $\left.\mathrm{m}^{2} \mathrm{IQR} 25.8-91.5 ; P=0.0002\right)$. $\mathrm{CD} 138-\operatorname{IgA}+$ and $\mathrm{CD} 138-\operatorname{IgG}+$ cells were also identified. The presence of ASCs could explain the observed Ig of dermal explant cultures (Figure 2).

\section{The foreskin epidermis permits the passage of selected} antibody isotypes

Ideally, vaccine-induced protection via HIV-1-specific antibodies would rely on antibodies present at sites of exposure and early infection, to prevent rapid initiation of local infection and spread. ${ }^{35-38}$ Thus, we explored the extent to which antibodies can permeate the foreskin epithelial barrier (Figure 2). All antibody isotypes had significantly lower Ig/HSA ratios in the outer epidermis than in the outer dermis $(P<0.014$; Figure 2). For example, median IgM in the outer epidermis was fourfold lower compared with the outer dermis $(P<0.001$; Figure 3f), median IgA was threefold lower $(P<0.001$; Figure 2a), and median IgG1 was twofold lower $(P<0.010$; Figure $2 \mathbf{b})$. Even more dramatic examples of reduced antibody transport across the epithelium were IgG2, IgG4, and IgE, as these isotypes were often undetectable at the epidermis (Figure 2c, e, g). The results indicated that the outer epidermal barrier restricts the passage of antibody isotypes to the most outer layers.

The inner foreskin epidermis, however, showed differences in permeability for the different isotypes (Figure 2). $\operatorname{IgA} \operatorname{IgM}$, $\mathrm{IgE}$, and IgG4 showed significant reductions in inner epidermis Ig/HSA ratios when compared with those in the inner dermis. For example, median IgM/HSA ratios in the inner epidermis were fivefold lower than those of the inner dermis; $(P<0.001$; Figure 3f). IgA/HSA ratios in the inner epidermis were fourfold reduced compared with the outer dermis $(P<0.001$; Figure 2a). This indicated that the epidermal barrier limits the passage of some isotypes.

Interestingly, inner foreskin epidermis IgG1, IgG2, and IgG3 ratios were not distinguishable from inner foreskin dermal levels (Figure 2c-e). These results suggested some preferential Ig deposition at the inner epidermis.

\section{Adenovirus 5 (Ad5) antibody responses at the foreskin come primarily from blood and can reach the inner foreskin epidermis}

To determine whether our methods would be useful to elucidate the distribution of antigen-specific responses at the foreskin, we used an Ad5 ELISA to assess the IgG/HSA and IgA/HSA ratios 
a

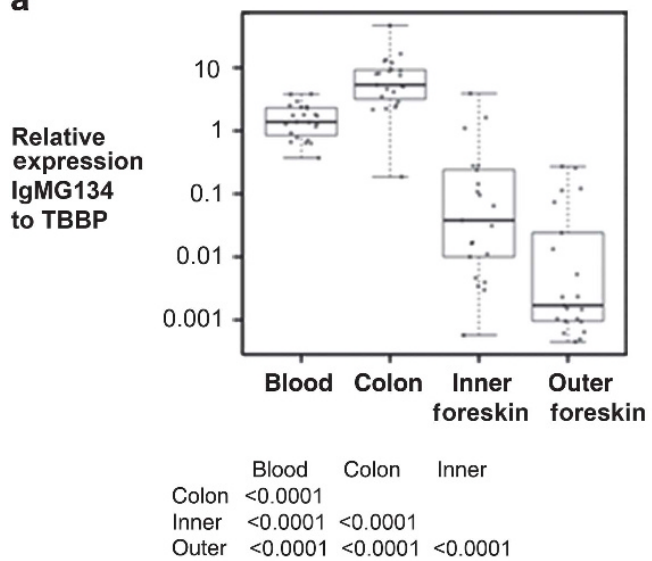

C

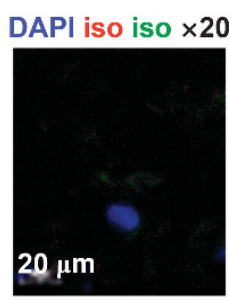

Outer foreskin

DAPI IgG CD138 ×20

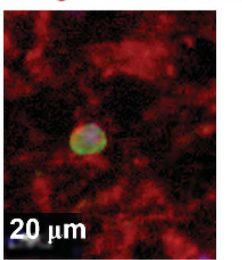

d

Outer foreskin

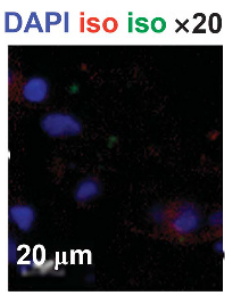

b

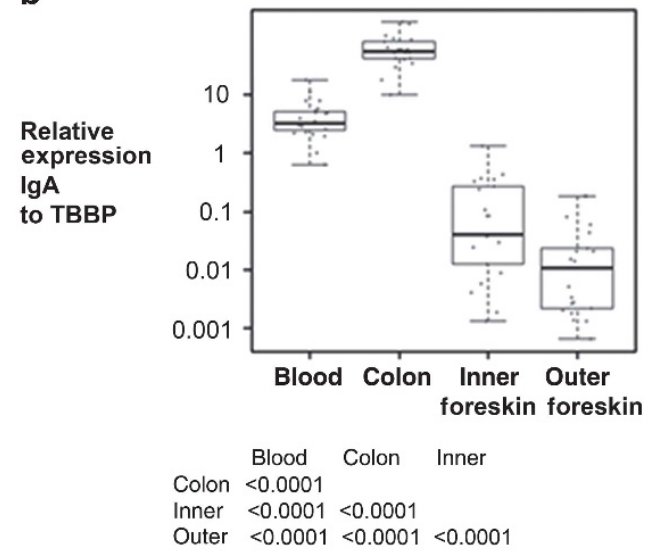

Inner foreskin

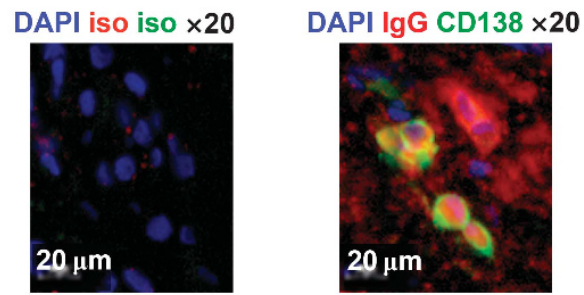

Inner foreskin

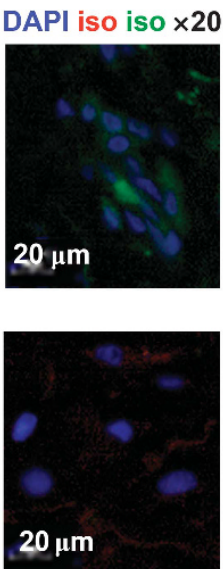

DAPI IgA CD138 ×20
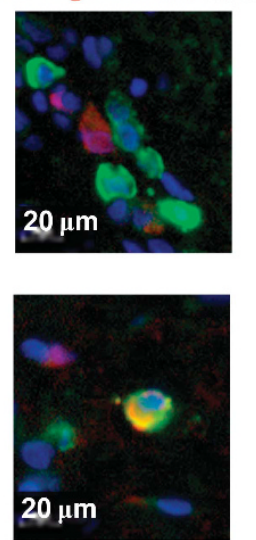

Figure 4 Foreskin tissue contains antibody secreting cells. (a, b) Real-time PCR comparing 20 paired colonic biopsies, blood, and inner and outer foreskin was used to quantitate mRNA transcription with a primer that amplifies (a) IgM, IgG1, IgG3, and IgG4 (IgMG134); and (b) IgA; normalized to TBBP. Only significant differences in Wilcoxon post-test after Bonferroni correction for six comparisons are reported beneath each graph. Individual measurements are represented by circles. Box plots show the median and IQR, with whiskers extending to the range of the data for each tissue. (c) Representative $20 \times$ magnification immunofluorescence microscopy images of paired inner and outer foreskin sections stained with DAPI nuclear counterstain (pseudocolored blue), CD138 (pseudocolored green), and IgG or their isotype control (both pseudocolored red). (d) Selected $20 \times$ magnification immunofluorescence microscopy images of paired inner and outer foreskin sections stained with DAPI nuclear counterstain (pseudocolored blue), CD138 (pseudocolored green), and IgA or their isotype control (pseudocolored red). In (c) and (d), we analyzed $\sim 2.6-\mathrm{mm}^{2}$ sections from 18 individuals, but photos represent only 1/200 of the area used to quantitate ASCs. ASCs, antibody-secreting cells; DAPI, 4',6-diamidino2-phenylindole; Ig, immunoglobulin; IQR, interquartile range; TBBP, TATA-box binding protein.

in plasma, colon, and foreskin explant supernatants using samples from the 14 Ad5-seropositive participants (Ad5 neutralization titer $>18$ ).

In colonic biopsies, Ad5-specific IgG responses were not distinguishable from those in plasma taken previous to the sigmoidoscopy $(P=0.0590$; Figure 5a). However, colonic
Ad5-specific IgA responses were 22-fold higher than those in plasma $(P<0.0001$; Figure $5 \mathbf{b})$. Thus, natural infection with adenovirus can generate abundant antibody responses in the colonic mucosa.

Contrary to our findings for total foreskin Ig (Figure 2), Ad5 IgG responses in plasma were higher than those at the outer 

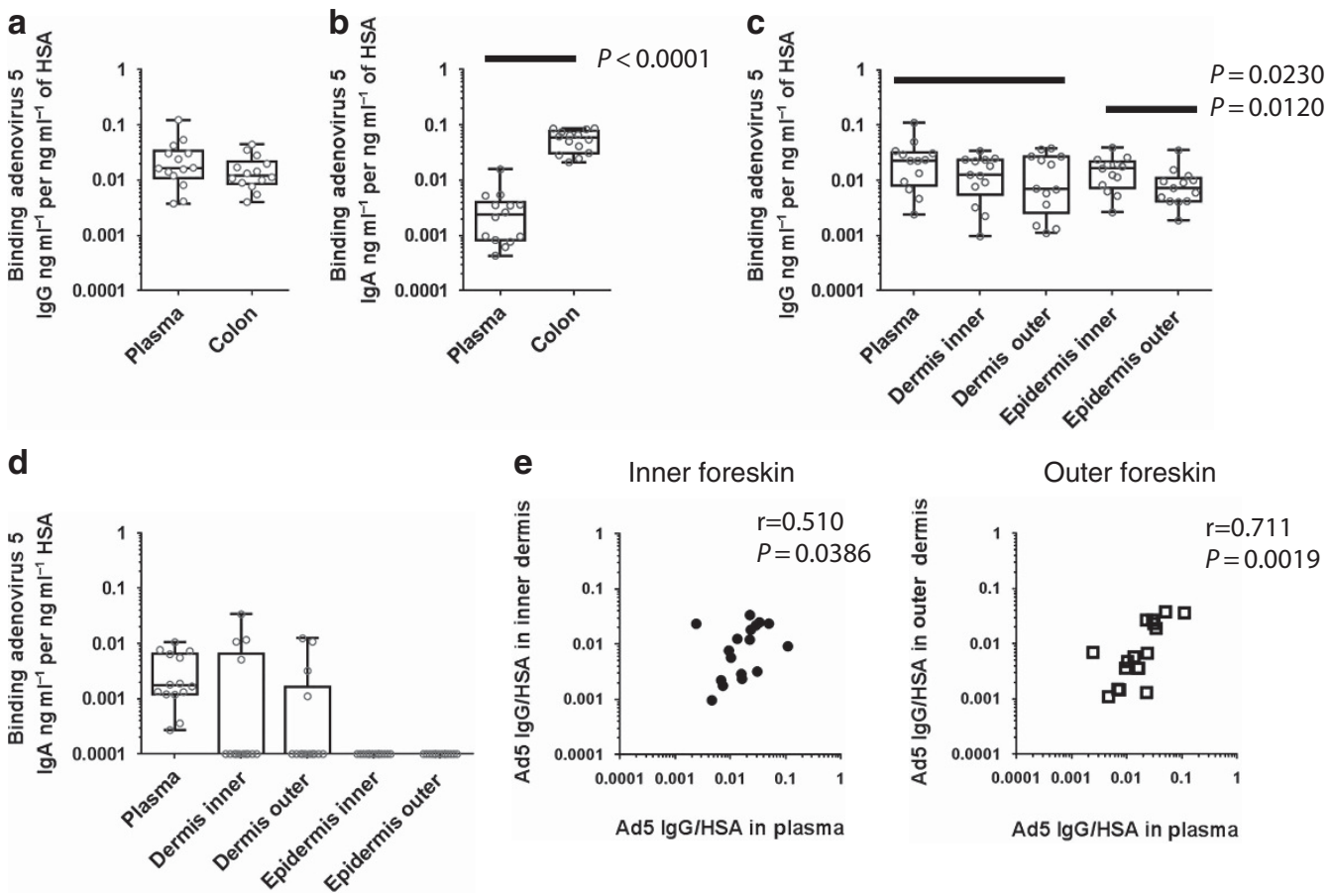

Figure 5 Foreskin Ad5 IgG transudates from blood and preferentially reaches the inner epidermis, whereas Ad5 IgA is mainly in the colon. (a-d) After exclusion of 6 samples owing to dermal contamination or Ad5-seronegative status, colon (a, b), foreskin (c, d), and corresponding plasma samples from 14 participants were compared by calculating the ratios of binding Ad5 $\lg G(\mathbf{a}, \mathbf{c})$ or $\lg A(\mathbf{b}, \mathbf{d})$ normalized to HSA in each sample. HSA was measured by MBA; Ad5 IgG and IgA was assessed by ELISA. Only significant differences in Wilcoxon post-test after Bonferroni correction for multiple comparisons are reported in the figures. (e) Correlation between 14 foreskin dermal Ad5 IgG/HSA ratios and those in paired blood were analyzed using Spearman's rho. Inner dermis samples are labeled with filled black circles; outer dermis samples are labeled with open black squares. Ad5, Adenovirus 5; Ig, immunoglobulin; HSA, human serum albumin; MBA, multiplex bead array.

foreskin dermis $(P=0.0230$; Figure $5 \mathbf{c})$. Ad5 IgG in plasma also trended higher than that in the inner dermis, but was not significantly different after adjustment for multiple comparisons. A similar pattern was observed when Ad5 IgA was compared (Figure 5d). These results indicated that local adenovirus IgG and IgA responses did not accumulate at the foreskin.

We also examined the correlation between dermal and plasma Ad5 IgG/HSA ratios (Figure 5e). Ad5 IgA ratios were not correlated, because many foreskin samples were below the limit of detection of the assay (Figure 5d). Both inner $(r=0.510$, Spearman's $P=0.0386)$ and outer $(r=0.711$, Spearman's $P=0.0019$ ) dermal Ad5 IgG ratios were moderately correlated to plasma, indicating that Ad5 IgG antibodies at the foreskin mainly transudate from blood.

Lastly, we assessed the differences among inner and outer foreskin epidermis Ad5 IgG/HSA ratios to evaluate our findings of differential antibody permeability in these surfaces (Figure 5c). Inner epidermis (median 0.016, IQR 0.007-0.022) had twofold more Ad5 IgG than outer epidermis (median 0.007, IQR 0.004-0.011; $P=0.0120$ ). This indicated that the inner foreskin can allow for increased penetration of Ad5 IgG antibodies.

\section{DISCUSSION}

We investigated the antibody isotypes present in different areas of the human foreskin and compared them with colonic mucosa and blood. Our results indicate that foreskin humoral responses differ from intestinal humoral responses in critical aspects such as the lack of inductive follicles, relatively few ASCs, and the relative frequency of specific Ig isotypes (such as increased IgG2 and reduced IgA). After $48 \mathrm{~h}$ of culture, IgA, IgG1, IgG3, and IgM Ig/HSA ratios at the foreskin epidermis were indistinguishable from blood ratios, but IgG2, IgG4, and IgE were rare. Thus, vaccine-induced immunity against HIV in males may need to meet distinct requirements in order to provide protection at systemic, rectal, and penile infection sites.

We explored whether the humoral immune response at the foreskin relies on diffusion/convection of systemic antibodies. In addition to blood sources, we found evidence that the foreskin contains local ASCs that may contribute to local IgM, IgA, IgG2, and IgE responses, as shown by increased dermal total Ig/HSA ratios compared with blood, the presence of Ig transcripts at the foreskin, the lack of correlation between inner dermal responses and blood, and the presence of dermal cells with intracellular IgA or IgG. Our results indicate that the foreskin combines roles for systemic responses and resident ASCs, similar to what has previously been shown for the intestine, the female reproductive tract, and seminal fluid. ${ }^{24-27}$ Thus, examining localized responses could reveal new mucosal and genital correlates of exposure and protection, such as the newly identified neutralizing anti-HIV IgA in subpreputial swabs. ${ }^{39,40}$

Examining Ig/HSA ratios also may aid understanding of the location of pathogen-specific responses, such as for adenovirus. 
Previous reports have indicated adenoviruses could be shed rectally in HIV-infected individuals; ${ }^{41}$ and uncircumcised Ad5-seropositive men who have sex with men vaccinated in the Step study were found to be at an increased risk of HIV infection. ${ }^{42}$ We show that Ad5-seropositive men who have sex with men have Ad5 IgG and IgA localized in the colonic mucosa, whereas only Ad5 IgG reaches the foreskin. These results suggest that natural adenovirus exposure does not attract Ad5 ASCs to this genital surface, but Ad5 IgG from plasma can reach dermal and epidermal compartments. Detecting antigen-specific antibodies in explant cultures may serve to evaluate whether adoptive transfer therapies and HIV vaccinations can reach the foreskin in humans, and provide a picture of the effector response at male genital sites.

Our data indicate that the foreskin has selective barriers for antibody transport at the epidermis. We demonstrate that the foreskin epithelium is relatively impermeable to IgG2, IgG4, and $\operatorname{IgE}$ transport, whereas $\operatorname{IgA} \operatorname{IgG} 1, \operatorname{IgG} 3$, and $\operatorname{IgM}$ are consistently detected at the foreskin epidermal layers. This could suggest differential turnover of the isotypes in the epithelium, or selective receptor-mediated transport. The polymeric Ig receptor could have a role in IgA and IgM movement across the epithelium, like it does in the gut and female genital compartments. ${ }^{43,44}$ IgG1 and IgG3 have higher affinity for the neonatal Fc receptor than IgG2 and IgG4, and it has been shown to modify IgG transport at the penile urethra and human endocervix. ${ }^{45}$ Future work will aim to elucidate the transport mechanisms, and define whether they can aid in control of infections at the foreskin.

In addition, our study is consistent with non-human primate studies, where the foreskin-localized B-cell response was concentrated at the inner foreskin. ${ }^{46}$ Compared with the outer layers, we observed increased transcription of local Igs at the inner foreskin. Blood and dermal Ig/HSA ratios for less dominant isotypes were directly correlated only at the outer foreskin, suggesting that local responses have a more important role at the inner foreskin This might correspond to a localized response to the inner foreskin microbiota, ${ }^{47}$ and may include localized antibody deposition from penile secretions, which can collect in the subpreputial space.

Given the enrolment restrictions of our study population, it is unclear whether these findings are common to all uncircumcised men, or whether it only represents young men at high HIV risk (with or without asymptomatic infections). One report has identified CD138 + IgA + cells in the foreskin of one low risk Kenyan man, but they were lacking in another one. ${ }^{39}$ Further work will determine whether humoral vaccine-induced responses can reach these sites of host-pathogen interaction and potentially provide protection for all uncircumcised males.

Curiously, our data suggest that both foreskin IgG3 and IgG4 antibodies are derived primarily from blood, but only IgG3 can reach the foreskin epithelium. This finding is interesting in the context of the RV144 study, where circulating anti-Env IgG3 antibodies correlated with reduced risk of infection, whereas the anti-Env antibodies in the non-protective VAX003 study utilizing a similar regimen were predominantly $\operatorname{IgG} 4 .{ }^{4,5}$ In addition to the functional consequences associated with each isotype, ${ }^{5,30}$ their ability to reach genital surfaces in sufficient concentration and to find appropriate mucosal cells for effector mechanisms may contribute to vaccine efficacy.

In summary, we have demonstrated that both local and systemic antibodies contribute to the foreskin immune response. We show selective antibody isotype penetration to the foreskin epidermis, especially at the inner foreskin. The assays presented in this paper, and the recently reported collection of subpreputial swabs, ${ }^{39,40}$ may provide valuable information to assess penile antibody responses in vaccine studies protecting against STIs.

\section{METHODS}

Tissue and blood donation. We evaluated foreskin and colon biopsy samples collected in Lima, Peru, during HVTN 914. They correspond to $20 \mathrm{HIV}$-negative, 21-30-year-old men who have sex with men at high risk of HIV acquisition. ${ }^{48}$ All study participants provided written informed consent prior to HVTN 914 participation, and met safety criteria to undergo elective circumcision and sigmoidoscopy procedures, as approved by the relevant institutional review boards.

Participants received two Ringer's Lactate enemas to cleanse the intestine previous to collection of 29 sigmoid biopsies using a $3.3-\mathrm{mm}$ diameter cup forceps. Two weeks later, circumcisions were performed using the sleeve procedure after dorsal penile nerve block anesthesia. The inner foreskin was identified on the basis of the relative decrease in melanocytes compared with the outer. Procedures were carried out after confirming the absence of signs and symptoms of infection, but samples collected at the time of procedures indicated that 10 of these individuals were STI-positive, when tested for HSV-2 serology $(n=5)$, Chlamydia trachomatis in rectal swabs $(n=3)$ and urine $(n=1)$, and/or Neisseria gonorrhoeae PCR in rectal swabs $(n=3)$.

Protein lysate preparation. Colonic biopsies or foreskin pieces were frozen at $-80^{\circ} \mathrm{C}$ within $2 \mathrm{~h}$ of surgery. Tissue was homogenized in lysate buffer, ${ }^{49}$ and normalized to $200 \mu \mathrm{g} \mathrm{ml}^{-1}$ of protein as measured by bicinchoninic acid assay (Pierce, Rockford, IL). ${ }^{48}$

Plasma isolation. Paired blood samples were collected by venipuncture into acid citrate dextrose tubes a few hours before the procedures. Plasma was isolated by consecutive centrifugations: first at $300 \times g$ to separate plasma from cells, and then at $800-1,000 \times g$ to remove any heme-containing contaminant.

Foreskin explant cultures. Epidermal sheets from foreskin were separated from dermis after dispase digestion, then washed and cultured for $48 \mathrm{~h}^{48}$ As only the dermis is vascularized, hemoglobin ELISA (ICL, Portland, OR) in epidermal explant supernatants was used to assess dermal contamination. ${ }^{48}$

Multiplex bead array. Tissue lysates, explant culture supernatants, and plasma were assayed for Ig content using a multiplex human Ig isotyping kit, single-plex HSA kit, and single-plex human IgE kit, which used plastic beads measured in a Luminex platform (all from Millipore, Billerica, MA). Samples were diluted to be within the quantitative ranges of the standard curves. Tissue lysates were assayed at 1:2, 1:10, and 1:100 dilutions in $0.5 \times$ lysate buffer, which was also used to prepare standard curves, positive controls, and background controls. Explant supernatants were assayed undiluted, 1:2, 1:200, and 1:400 in RPMI 20\% fetal bovine serum; plasma samples were assayed at 1:30, 1:15,000, and 1:150,000 in RPMI 20\% fetal bovine serum, along with standard curves, positive controls, and background controls diluted in the same buffer. 
Curve fitting for multiplex bead array used the nCal R package. ${ }^{50}$ A conservative lower limit of detection was defined as the estimated concentration at which the lower confidence interval of the estimate equaled the lowest standard used in the calibration curve. All Ig values smaller than this limit were replaced by 0 . All samples contained HSA values within the standard curve limits of detection so there was no replacement for HSA.

Concentrations of antibody in tissue lysates were normalized to protein content to permit comparisons among foreskin and colon. Because explant cultures contained fetal bovine serum, normalization was not carried out with respect to protein concentration, but rather with HSA as it is synthesized in the liver and transported from blood through the skin via convection. ${ }^{51}$ This accounted for anticoagulant dilution in the plasma collections and explant tissue size in the cultures.

RNA isolation, reverse transcription, and quantitative PCR. Inner and outer foreskin pieces and colon biopsies were preserved in RNAlater (Life Technologies, Grand Island, NY) and stored at $-80^{\circ} \mathrm{C}$. RNA isolations were carried out by placing the thawed tissue in $1 \mathrm{ml}$ of TRI Reagent (Life Technologies) and homogenizing it with a homogenizer with wide probe (Omni, Kennesaw, GA). Nucleic acid separation used 1-bromo-3-chloropropane (Sigma-Aldrich, St. Louis, MO) and Ribopure Kit (Life Technologies) columns. Blood was collected in Tempus tubes, with RNA isolated according to the Tempus Spin RNA Isolation Kit (Life Technologies).

RNA integrity was measured with an Agilent 2100 Bioanalyzer (Agilent, Santa Clara, CA), and all samples had a median RNA integrity number of 8.9 (IQR 8.3-9.3), well above the necessary quality threshold to conduct these studies. One microgram of RNA was used in reverse transcriptions using Superscript VILO (Life Technologies).

RT-PCR samples containing 1 ng of the original RNA input were quantitated using Taqman Gene Expression assays (Life Technologies) designed to expand across exons within TBBP as an endogenous control, and a primer designed to amplify shared sequences in IgM, IgG1, IgG3, and IgG4 (IgMG134), or IgA. A 7900HT real-time PCR machine (Applied Biosystems, Foster City, CA) with 384-well plate handling was used to run each of the samples. A standard curve, containing $10^{8}$ to 5.65 copies of the specific PCR product cloned in a TOPO pCR2.1 plasmid (Life Technologies) was used to extrapolate absolute transcript copy numbers using an nCal R package. ${ }^{50}$ Three different blood samples from each of the procedure visits, three colonic samples from two sigmoidoscopy procedures, and three to five inner or outer foreskin samples were each run in duplicate and summarized by tissue for each participant using a trimmed mean to remove extreme outliers. The IgMG134 and IgA copies were normalized to TBBP expression, which was the least variable endogenous control when compared with $\beta$-actin, $\beta$-glucuronidase, and cyclophilin A among the different tissues.

Immunofluorescence microscopy. Foreskin pieces were fixed in buffered formalin for a maximum of 7 days, dehydrated in $70 \%$ ethanol, and paraffin-embedded. Four-micron-thick sections were deparaffinized and rehydrated through graded alcohols to distilled water for staining. Sections were incubated $20 \mathrm{~min}$ in pH6.0 Target Retrieval Solution (DakoCytomation, Carpinteria, CA), cooled, and loaded onto the DakoCytomation Autostainer. Endogenous peroxidases and biotin were blocked with $3 \% \mathrm{H}_{2} \mathrm{O}_{2}$, followed by a Avidin/ Biotin block (Biocare, Concord, CA) following manufacturer's instructions, and a $0.25 \%$ casein TBS, $0.1 \%$ Tween-20 (Sigma-Aldrich) solution. Anti-CD138 (1:25 from Cell Marque, Rocklin, CA) or mouse IgG isotype $\left(0.2 \mu \mathrm{g} \mathrm{ml}^{-1}\right)$ was added to the slide for $60 \mathrm{~min}$, followed by biotinylated anti-mouse IgG (1:200; Vector Laboratories, Burlingame, CA) and streptavidin Ax568 (Invitrogen, Carlsbad, CA) for $30 \mathrm{~min}$ each. For IgM/IgG staining, goat anti-human IgG and IgM (1:5,000; Jackson Immunoresearch, West Grove, PA) or goat isotype $\left(3.4 \mu \mathrm{g} \mathrm{ml}^{-1}\right)$ was added for $60 \mathrm{~min}$ followed by CSA II amplification for readout on fluorescein isothiocyanate. For IgA, rabbit anti-human
IgA $(1: 5,000)$ or isotype $\left(4.8 \mu \mathrm{g} \mathrm{ml}^{-1}\right)$ was added for $60 \mathrm{~min}$, washed, and stained with goat anti-rabbit Alexa Fluor 647 (1:50, Invitrogen) for $30 \mathrm{~min}$.

Samples were counterstained with 4',6-diamidino-2-phenylindole, coverslipped with ProLong Gold (Life Technologies), cured, and then stored at $4{ }^{\circ} \mathrm{C}$ until image acquisition. Slides were scanned on an Aperio FL (Leica Microsystems, Buffalo Grove, IL) using single-band filter cubes (Semrock, Rochester, NY) for each fluorochrome. Exposure times were determined from stained control tissue and remained constant on experimental samples. The validity of the staining was confirmed on colon, abdominal skin, and lymph node sections (data not shown).

Adenovirus 5 (Ad5) neutralization assay and ELISA. An Ad5 neutralization assay was carried out using participant plasma obtained 1 week before sigmoidoscopy as previously described. ${ }^{52}$

For ELISA, 96-well plates (Thermo Scientific, Waltham, MA) were coated overnight with $1 \times 10^{9}$ viral particles of Ad5 purified by doublebanding, CsCl gradients, and dialysis, and diluted in $100 \mu \mathrm{l}$ of coating buffer (Immunochemistry Technologies, Bloomington, MN). Plates were then blocked for $2 \mathrm{~h}$ with $5 \%$ non-fat milk (Fisher, Scientific, Hampton, NH), 3\% Inactivated Goat Serum (Invitrogen), and $0.2 \%$ Tween-20 (Sigma-Aldrich) in $1 \times$ phosphate-buffered saline (Life Technologies). Explant samples were assayed undiluted and at 1:10 and 1:100 dilutions in blocking buffer, and negative controls included RPMI with $20 \%$ fetal bovine serum. Plasma samples were diluted in six 1:5 dilutions starting with 1:500. Samples, standard curves, and controls were incubated for $2 \mathrm{~h}$ at $37^{\circ} \mathrm{C}$.

After washing, goat anti-human IgG-HRP (Southern Biotech, Birmingham, AL) or mouse anti-human IgA-HRP (Abcam, Cambridge, MA) at 1:1,000 was incubated for $1 \mathrm{~h}$ at $37^{\circ} \mathrm{C}$, then detected with addition of BM Blue substrate (Roche, Basel, Switzerland) for $15 \mathrm{~min}$. The reaction was stopped by adding $50 \mu \mathrm{l}$ of $1 \mathrm{~N} \mathrm{H}_{2} \mathrm{SO}_{4}$ and read within 20 min using a SpectraMax M2 ELISA Plate Reader (Molecular Devices, Sunnyvale, CA) at $450 \mathrm{~nm}$ absorbance. Standard curves used human IgG (Bethyl Laboratories, Montgomery, TX) or human IgA (Sigma-Aldrich), at 20.0 to $0.009 \mathrm{ng}$ per well, and the linear ranges were used for extrapolation of the concentrations.

Statistical analysis. Statistical comparisons were carried out using Prism v6 (GraphPad Software, San Diego, CA). Nonparametric comparisons among multiple tissues were carried out using the Friedman test. ${ }^{53}$ If significant, a Wilcoxon signed ranked post-test was used to identify differences between two specific tissues. Unless specifically described, all $P$ values described are Wilcoxon $P$ values corrected for multiple comparisons using Bonferroni. Spearman's correlation was used to assess the association between dermal antibody HSA ratios and those in paired blood. Only comparisons with $r>0.5$ and $P<0.05$ were considered significant.

\section{ACKNOWLEDGMENTS}

We thank the study volunteers for their participation; Dr V.M. Barrientos, $\mathrm{Dr} \mathrm{H}$. Agurto, and the clinical staff at IMPACTA for follow-up of study participants; Matt Perez, Tracy Goodpaster, Jane Vasylyeva, Greg Spies, Maria E. Gamero, Sonia Minaya, Richard Negrón, Jesus Jurupe, Monica Nieto, Nilda Gadea, Elisa Ahern, and McKenzie Momany for laboratory support. We thank the FHCRC Genomics, Imaging Laboratory, and Experimental Histopathology Cores for immunofluorescence microscopy assistance and Agilent measurements. We thank Florian Hladik, Georgia Tomaras, and Julie Overbaugh for helpful discussion, and Stephen Voght for critical reading of the manuscript. We thank Gina Escamilla and Cheryl de Boer for help with data management, and Sarah Holte for consultations at the CFAR Biostatistical Core. We thank the James B. Pendleton Charitable Trust for their generous equipment donation. Research reported in this publication was supported by the National Institute of Allergy and Infectious Disease of the National Institutes of Health under awards numbers UM1AI068618, UM1AI068614, U01Al069438 and a New Investigator Award from the University of Washington Center for AIDS Research (CFAR) P30AI027757. The content is solely the responsibility of the authors 
and does not necessarily represent the official views, policy or position of the National Institutes of Health, Department of the Navy, Department of Defense, or U.S. Government. Dr Montano is an employee of the U.S. Government who contributed to this work as part of her official duties. No copyright protection is available for her work as defined by Titles 17 U.S.C. $\S 105$ and $\$ 101$.

\section{DISCLOSURE}

The authors declare no conflict of interest.

(c) 2016 Society for Mucosal Immunology

\section{REFERENCES}

1. Lynch, R. M., Yamamoto, T. \& McDermott, A. B. HIV vaccine research and discovery in the nonhuman primates model: a unified theory in acquisition prevention and control of SIV infection. Curr. Opin. HIV AIDS 8, 288-294 (2013).

2. Rerks-Ngarm, S. et al. Vaccination with ALVAC and AIDSVAX to prevent HIV-1 infection in Thailand. N. Engl. J. Med. 361, 2209-2220 (2009).

3. Haynes, B. F. et al. Immune-correlates analysis of an HIV-1 vaccine efficacy trial. N. Engl. J. Med. 366, 1275-1286 (2012).

4. Yates, N. L. et al. Vaccine-induced Env V1-V2 lgG3 correlates with lower HIV-1 infection risk and declines soon after vaccination. Sci. Transl. Med. 6 , 228ra239 (2014).

5. Chung, A. W. et al. Polyfunctional Fc-effector profiles mediated by lgG subclass selection distinguish RV144 and VAX003 vaccines. Sci. Transl. Med. 6, 228ra238 (2014).

6. Goedert, J. J. et al. Determinants of retrovirus (HTLV-III) antibody and immunodeficiency conditions in homosexual men. Lancet 2, 711-716 (1984).

7. Johnson, A. M. et al. Transmission of HIV to heterosexual partners of infected men and women. AIDS 3, 367-372 (1989).

8. Mestecky, J. Humoral immune responses to the human immunodeficiency virus type-1 (HIV-1) in the genital tract compared to other mucosal sites. J. Reprod. Immunol. 73, 86-97 (2007).

9. Auvert, B. et al. Randomized, controlled intervention trial of male circumcision for reduction of HIV infection risk: the ANRS 1265 Trial. PLoS Med. 2, e298 (2005).

10. Gray, R. H. et al. Male circumcision for HIV prevention in men in Rakai, Uganda: a randomised trial. Lancet 369, 657-666 (2007).

11. Bailey, R. C. et al. Male circumcision for HIV prevention in young men in Kisumu, Kenya: a randomised controlled trial. Lancet 369, 643-656 (2007).

12. Sanchez, J. et al. Male circumcision and risk of HIV acquisition among MSM. AIDS 25, 519-523 (2011).

13. Goodreau, S. M. et al. What drives the US and Peruvian HIV epidemics in men who have sex with men (MSM)? PLoS One 7, e50522 (2012).

14. Dinh, M. H. et al. Visualization of HIV-1 interactions with penile and foreskin epithelia: clues for female-to-male HIV transmission. PLoS Pathog. 11, e1004729 (2015).

15. UNAIDS, W. A. New Data on Male Circumcision and HIV Prevention: Research Implications for Policy and Programming. WHO and UNAIDS, Montreux, Switzerland (2007).

16. Auvert, B. et al. Estimating the resources needed and savings anticipated from roll-out of adult male circumcision in Sub-Saharan Africa. PLoS One 3, e2679 (2008)

17. Hallett, T. B. et al. Understanding the impact of male circumcision interventions on the spread of HIV in southern Africa. PLoS One 3, e2212 (2008).

18. Herman-Roloff, A., Otieno, N., Agot, K., Ndinya-Achola, J. \& Bailey, R. C. Acceptability of medical male circumcision among uncircumcised men in Kenya one year after the launch of the national male circumcision program. PLoS One 6, e19814 (2011).

19. Tauber, P. F., Zaneveld, L. J., Propping, D. \& Schumacher, G. F. Components of human split ejaculates. I. Spermatozoa, fructose, immunoglobulins, albumin, lactoferrin, transferrin and other plasma proteins. J. Reprod. Fertil. 43, 249-267 (1975).

20. Rumke, P. The origin of immunoglobulins in semen. Clin. Exp. Immunol. 17, 287-297 (1974).
21. Gelzayd, E. A., Kraft, S. C. \& Kirsner, J. B. Distribution of immunoglobulins in human rectal mucosa. I. Normal control subjects. Gastroenterology 54 , 334-340 (1968).

22. Macpherson, A. J., McCoy, K. D., Johansen, F. E. \& Brandtzaeg, P. The immune geography of IgA induction and function. Mucosal Immunol. 1, 11-22 (2008).

23. Brandtzaeg, P., Baklien, K., Fausa, O. \& Hoel, P. S. Immunohistochemical characterization of local immunoglobulin formation in ulcerative colitis. Gastroenterology 66, 1123-1136 (1974).

24. Crago, S. S. et al. Distribution of IgA1-, IgA2-, and J chain-containing cells in human tissues. J. Immunol. 132, 16-18 (1984).

25. Kutteh, W. H., Prince, S. J., Hammond, K. R., Kutteh, C. C. \& Mestecky, J. Variations in immunoglobulins and IgA subclasses of human uterine cervical secretions around the time of ovulation. Clin. Exp. Immunol. 104, 538-542 (1996).

26. Moldoveanu, Z., Huang, W. Q., Kulhavy, R., Pate, M. S. \& Mestecky, J. Human male genital tract secretions: both mucosal and systemic immune compartments contribute to the humoral immunity. J. Immunol. 175, 4127-4136 (2005).

27. Pudney, J. \& Anderson, D. J. Immunobiology of the human penile urethra. Am. J. Pathol. 147, 155-165 (1995).

28. Bjercke, S. \& Brandtzaeg, P. Glandular distribution of immunoglobulins, J chain, secretory component, and HLA-DR in the human endometrium throughout the menstrual cycle. Hum. Reprod. 8, 1420-1425 (1993).

29. Kutteh, W. H., Hatch, K. D., Blackwell, R. E. \& Mestecky, J. Secretory immune system of the female reproductive tract: I. Immunoglobulin and secretory component-containing cells. Obstet. Gynecol. 71, 56-60 (1988).

30. Bruhns, P. et al. Specificity and affinity of human Fcgamma receptors and their polymorphic variants for human IgG subclasses. Blood 113, 37163725 (2009).

31. Kantele, A. et al. Differences in immune responses induced by oral and rectal immunizations with Salmonella typhi Ty21a: evidence for compartmentalization within the common mucosal immune system in humans. Infect. Immun. 66, 5630-5635 (1998).

32. Lubeck, M. D. et al. Immunogenicity of recombinant adenovirus-human immunodeficiency virus vaccines in chimpanzees following intranasal administration. AIDS Res. Hum. Retroviruses 10, 1443-1449 (1994).

33. Yang, O. O. et al. Differential blood and mucosal immune responses against an HIV-1 vaccine administered via inguinal or deltoid injection. PLoS One 9, e88621 (2014).

34. Kutteh, W. H. \& Mestecky, J. Secretory immunity in the female reproductive tract. Am. J. Reprod. Immunol. 31, 40-46 (1994).

35. Mehandru, S. et al. Mechanisms of gastrointestinal CD4 + T-cell depletion during acute and early human immunodeficiency virus type 1 infection. J. Virol. 81, 599-612 (2007)

36. Hel, Z., McGhee, J. R. \& Mestecky, J. HIV infection: first battle decides the war. Trends Immunol. 27, 274-281 (2006).

37. Li, Q. et al. Visualizing antigen-specific and infected cells in situ predicts outcomes in early viral infection. Science 323, 1726-1729 (2009).

38. Hladik, F. et al. Initial events in establishing vaginal entry and infection by human immunodeficiency virus type-1. Immunity 26, 257-270 (2007).

39. Hirbod, T. et al. HIV acquisition is associated with increased antimicrobial peptides and reduced HIV neutralizing IgA in the foreskin prepuce of uncircumcised men. PLoS Pathog. 10, e1004416 (2014).

40. Prodger, J. L. et al. Immune correlates of HIV exposure without infection in foreskins of men from Rakai, Uganda. Mucosal Immunol. 7, 634-644 (2014).

41. Curlin, M. E. et al. Frequent detection of human adenovirus from the lower gastrointestinal tract in men who have sex with men. PLoS One 5, e11321 (2010).

42. Buchbinder, S. P. et al. Efficacy assessment of a cell-mediated immunity HIV-1 vaccine (the Step Study): a double-blind, randomised, placebocontrolled, test-of-concept trial. Lancet 372, 1881-1893 (2008).

43. Kaetzel, C. S., Robinson, J. K., Chintalacharuvu, K. R., Vaerman, J. P. \& Lamm, M. E. The polymeric immunoglobulin receptor (secretory component) mediates transport of immune complexes across epithelial cells: a local defense function for IgA. Proc. Natl Acad. Sci. USA 88, 8796-8800 (1991).

44. Natvig, I. B., Johansen, F. E., Nordeng, T. W., Haraldsen, G. \& Brandtzaeg, P. Mechanism for enhanced external transfer of dimeric IgA over 


\section{ARTICLES}

pentameric IgM: studies of diffusion, binding to the human polymeric Ig receptor, and epithelial transcytosis. J. Immunol. 159, 4330-4340 (1997).

45. Gupta, S. et al. The neonatal $F_{C}$ receptor ( $\left.F_{C} R n\right)$ enhances human immunodeficiency virus type 1 (HIV-1) transcytosis across epithelial cells. PLoS Pathog. 9, e1003776 (2013).

46. Rothaeusler, K. et al. Antiviral antibodies and T cells are present in the foreskin of simian immunodeficiency virus-infected rhesus macaques. J. Virol. 86, 7098-7106 (2012).

47. Price, L. B. etal. The effects of circumcision on the penis microbiome. PLoS One 5, e8422 (2010).

48. Lemos, M. P. et al. The inner foreskin of healthy males at risk of HIV infection harbors epithelial CD4 + CCR5 + cells and has features of an inflamed epidermal barrier. PLoS One 9, e108954 (2014).
49. Hendrix, S. W. et al. Optimization of the skin multiple analyte profile bioanalytical method for determination of skin biomarkers from D-Squame tape samples. Skin Res. Technol. 13, 330-342 (2007).

50. Fong, Y., Sebestyen, K., Yu, X., Gilbert, P. \& Self, S. nCal: a R package for nonlinear calibration. Bioinformatics 29, 2653-2654 (2013).

51. Rothschild, M. A., Bauman, A., Yalow, R. S. \& Berson, S. A. Tissue distribution of 1131 labeled human serum albumin following intravenous administration. J. Clin. Invest. 34, 1354-1358 (1955).

52. Aste-Amezaga, M. et al. Quantitative adenovirus neutralization assays based on the secreted alkaline phosphatase reporter gene: application in epidemiologic studies and in the design of adenovector vaccines. Hum. Gene Ther. 15, 293-304 (2004).

53. Hollander, M., Wolfe, D. A. \& Chicken, E. Nonparametric Statistical Methods, 3rd edn. (Wiley, Hoboken, NJ, 2013). 